\title{
Cylinders through Five Points: Computational Algebra and Geometry
}

\author{
Daniel Lichtblau ${ }^{1}$ \\ ${ }^{1}$ Wolfram Research, Inc., 100 Trade Center Dr., Champaign, IL, USA \\ Correspondence: Daniel Lichtblau, Wolfram Research, Inc., 100 Trade Center Dr., Champaign, IL 61820, USA. \\ E-mail: danl@wolfram.com
}

Received: August 14, 2012 Accepted: October 19, 2012 Online Published: November 26, 2012

doi:10.5539/jmr.v4n6p65 URL: http://dx.doi.org/10.5539/jmr.v4n6p65

\begin{abstract}
We address the following question: Given five points in $\mathbb{R}^{3}$, determine a right circular cylinder containing those points. We obtain algebraic equations for the axial line and radius parameters and show that these give six solutions in the generic case. An even number $(0,2,4$, or 6$)$ will be real valued and hence correspond to actual cylinders in $\mathbb{R}^{3}$. We will investigate computational and theoretical matters related to this problem. In particular we will show how exact and numeric Gröbner bases, equation solving, and related symbolic-numeric methods may be used to advantage. We will also discuss some applications.
\end{abstract}

Keywords: computational geometry, enumerative geometry, Gröbner bases, nonlinear systems, symbolic-numeric computation

\section{Outline of the Problem and Related Work}

Given five points in $\mathbb{R}^{3}$, we are to determine all right circular cylinders containing those points. We do this by solving equations for the axial line and radius parameters. We will show that generically one obtains six solutions to these equations. Of these an even number are real valued, as the complex valued ones appear in conjugate pairs (an immediate consequence is that there is no "unique" real cylinder through five given points unless it a solution with multiplicity). Moreover there are open regions in the real configuration space that give each of these possibilities so none are disallowed.

The basic problem of determining cylinders from five points may be recast in a computational geometry setting: Given five points in $\mathbb{R}^{3}$, find the smallest positive $r$ and orientation parameters such that the cylinder of radius $2 r$ with those parameters encloses tangentially the balls of radius $r$ centered at the points.

Here are some questions we will consider. The first three are classical; we address them here to illustrate the utility of symbolic computation in such investigations. The last ones are related to more recent work in computational and integral geometry.

(1) Given the points and corresponding cylinder parameters, how might we display them graphically?

(2) Given the cylinder parameters, how may we obtain its implicit equation as a hypersurface in $\mathbb{R}^{3}$ ?

(3) Reversing this, how can one obtain parameters from the implicit form?

(4) Given five points chosen with random uniform distribution in a cube, what is the expected probability that one lies inside the convex hull of the other four (this is related to the "no real cylinder" case).

(5) How might we rigorously provide, via straightforward computation, the generic number of solutions to the algebraic equations that describe cylinders through five indeterminate points.

(6) Given six or more points, how do we find the coordinates of a (generically unique) cylinder in $\mathbb{R}^{3}$ that "best" fits those points?

(7) Given six or more points, how do we find the cylinder(s) of smallest radius enclosing them?

The problem of finding cylinders through five points may be recast in a computational geometry setting: Given five points in $\mathbb{R}^{3}$, find the smallest positive $r$, and corresponding orientation parameters. such that the cylinder of radius $2 r$ with those parameters encloses tangentially the balls of radius $r$ centered at the points. The cylinder axis will 
touch all the spheres tangentially. The coaxial cylinder of radius $r$ will exactly contain the five points. There are also related ways of viewing the problem of finding cylinders of a given radius and containing four specified points (Macdonald, Pach, \& Theobald, 2001). The last two items above also have important applications in metrology (see references below).

In the sequel we frequently use the term "real cylinders" to denote real valued solutions to the cylinder equations that arise from a given configuration of five points. Sometimes we refer to arbitrary solutions as "cylinders" even if they have complex values. The meaning should be clear from context. We refer to configurations as "generic" if they do not have multiple solutions and if all sufficiently small perturbations of the configuration give rise to the same number of solutions. This amounts to the configuration not lying on the discriminant variety (Lazard \& Rouillier, 2007). In some places we also use generic to mean that a system is in general position so that the Shape Lemma applies (Becker, Marinari, Mora, \& Traverso, 1994). As we will have occasion to change our underlying set of variables we note that this last notion is dependent on the variables under consideration.

That one obtains six cylinders was previously demonstrated in (Bottema \& Veldkamp, 1977) though by rather different means. Various proofs are also presented in (Chaperon \& Goulette, 2003; Devillers, Mourrain, Preparata, $\&$ Trebuchet, 2003; Lichtblau, 2007). A related problem, finding cylinders of a given radius through four given points in $\mathbb{R}^{3}$, is discussed in (Durand, 1998; Hoffmann \& Yuan, 2000; Schömer, Sellen, Teichmann, \& Yap, 2000; Macdonald et al., 2001). A nice survey of computational commutative algebra methods that are applicable to nonlinear (2007) problems in computational geometry can be found in (Buchberger, 1988). Another good general treatment of theoretical and practical aspects of Gröbner bases in computational geometry is chapter 7 of (Hoffmann, 1989). An earlier paper (Lichtblau, 2007), in many ways a companion to this one, delves into enumerative geometry aspects of cylinders through five points. The present paper, in contrast, will expose the many computational tools the author has developed in the process of investigating this family of cylinder problems. While computation is the emphasis herein we will, along the way, also recover several nontrivial results from the literature (Brandenberg \& Theobald, 2004; Chaperon \& Goulette, 2003; Devillers et al., 2003; Lichtblau, 2007; Petitjean, 2012). We also prove a new result that was conjectured in (Lichtblau, 2007). Moreover we provide a counterexample to a conjecture from (Devillers et al., 2003). Specifically, we provide a set of four points through which there are 14 real cylinders of extremal radius; the conjecture was that there could be at most 12 such extremal cylinders.

The remainder of this paper is structured as follows. In section 2 we present several computational sides to the problem. These include finding and counting cylinder solutions as well as standard topics arising from nonlinear computational geometry. In section 3 we handle the various associated computational geometry problems, and basics of point/cylinder visualization. Section 4 delves into the frequencies of real cylinders containing random point sets from a certain distribution. These investigations are again largely computational, though we relate some to a recent result in integral geometry (Zinani, 2003). In section 5 we use simple symbolic computation methods to prove the enumerative geometry result that there are six solutions to a generic set of cylinder equations. We also work with cylinders through four points, of either extremal or fixed radius, using similar techniques. Section 6 poses some further questions regarding cylinders through five points, and proves a conjecture from (Lichtblau, 2007) about the case of an infinite solution set. Following that is a brief summary. An attempt is made to emphasize the ways in which symbolic, numeric, and hybrid computation methods are useful in these investigations. Computations in the sequel were performed with version 8 of Mathematica (Wolfram, 2010); implementation code is available from the author.

\section{Computing Cylinders through Five Points}

\subsection{Finding Cylinder Parameters from a Set of 5 Points}

We will assume unless otherwise stated that our points are generic. In particular we will assume the following.

(1) No three points are collinear and no four are coplanar.

(2) Cylinder axes are not parallel to coordinate planes (in the formulation below we really only require that they not be parallel to the $y z$ coordinate plane). This will allow us to parametrize the axial direction as $(1, a, c)$ and the offset vector as $(0, b, d)$.

With these assumptions we avoid computational pitfalls that would arise from parametrizing axial directions using a sphere (this gives rise to two problems: we have one extra variable, and so to eliminate it we would add an equation that normalizes the direction. Moreover we would double the size of our solution set because any direction is 
equivalent to its negative). We remark that, in situations where points give cylinders with axes parallel to coordinate planes, a simple rotation of coordinates suffices to restore the required genericity. We note, moreover, that extensive computational experiments indicate this is never needed with (pseudo)random point configurations, insofar as they are always sufficiently far from the nongeneric bad configurations as to offer no numerical difficulty. The practical implication of this observation is that a random rotation of coordinates prior to subsequent computation will always suffice for computational purposes, even in situations where point placement might be pathological for our specific formulation. This applies both to configurations that lie in the algebraic set of nongeneric ones, and also to cases where the configuration is close to but not on that set and thus might thus cause numeric issues in computing via the original formulation.

Given these stipulations we proceed as follows. With our assumptions in place, given a cylinder axis line $L$ in $\mathbb{R}^{3}$ we may parametrize it as

$$
(y=a x+b, z=c x+d)
$$

For any $r>0$ there is a unique circular cylinder $C$ of radius $r$ with center axis $L$. Supposing we have five points on that cylinder the following questions now arise. How do we find $L$ and $r$ ? How do we use them to parametrize $C$ e.g. for purposes of plotting it?

First we discuss why this data will determine finitely many cylinders. Given a point on $C$ we will project orthogonally onto $L$ in order to get an equation involving the parameters we wish to find. We have five parameters to determine in the setup used above. For each point we denote the length of the orthogonal projection by $o_{j}$. It is computed as follows. We take $\tilde{L}$ to be the subspace obtained by translating $L$ to pass through the origin. For each point $p_{j}$ take $\tilde{p}_{j}$ to be the correspondingly translated point. We subtract from $\tilde{p}_{j}$ its projection onto $\tilde{L}$. This difference, $o_{j}$, is the orthogonal complement of the projection. This gives an algebraic equation of the form

$$
\left\|o_{j}\right\|^{2}-r^{2}=0
$$

A concise coordinate-free formulation of this appears in (Sottile, 2001). We show here the actual equation in terms of our point coordinates and cylinder parameters. If $p_{j}$ is given as $\left(x_{j}, y_{j}, z_{j}\right)$ then, after clearing denominators, the explicit equation in terms of cylinder parameters $(a, b, c, d, r)$ is:

$$
\begin{gathered}
a^{2} d^{2}-2 a^{2} d z_{j}+a^{2} x_{j}^{2}+a^{2} z_{j}^{2}-a^{2} r^{2}-2 a b c d+2 a b c z_{j}+2 a b x_{j}+2 a c d y_{j}-2 a c y_{j} z_{j}-2 a x_{j} y_{j}+b^{2} c^{2}+ \\
b^{2}-2 b c^{2} y_{j}-2 b y_{j}+c^{2} x_{j}^{2}+c^{2} y_{j}^{2}-c^{2} r^{2}+2 c d x_{j}-2 c x_{j} z_{j}+d^{2}-2 d z_{j}+y_{j}^{2}+z_{j}^{2}-r^{2}=0
\end{gathered}
$$

For generic choice of points the equations should be algebraically independent, hence the dimension of the solution set would be zero. In more detail, if we take five points with indeterminate coordinates (that is, coordinates expressed as variables) then we obtain a system of five equations of the form $f_{j}(a, b, c, d, r)=0$, each arising from (3) with appropriate point coordinates plugged in. From these we want to solve for the cylinder parameters in terms of those coordinates. To show there are finitely many solutions it suffices by the implicit function theorem to show that the Jacobian of the map $\left(f_{1}, f_{2}, f_{3}, f_{4}, f_{5}\right)$ has full rank for these generic coordinates. One can do this explicitly by finding the symbolic Jacobian, plugging in random values for the coordinates, and checking that the resulting matrix has full rank. We will instead show a computation in the last section that demonstrates there are generically at most nine solutions. Simple reasoning will further reduce this to eight. We also provide computational proofs that there are in fact only six.

Let us demonstrate how to solve for the cylinder parameters with a specific example. We will take as our parameter values

$$
a=3, b=2, c=4, d=-1, r=\sqrt{21}
$$

The locus of points on $C$ is obtained as sums of a vector on $L$ plus a vector of length $r$ perpendicular to $L$. All vectors perpendicular to $L$ are spanned by any independent pair. We can obtain an orthonormal pair $\left(w_{1}, w_{2}\right)$ in the standard way by finding the null space to the matrix whose one row is the vector along the axial direction, that is, $v=(1, a, c)$, and then using Gram-Schmidt to orthogonalize that pair. From this we obtain vectors $(-4 / \sqrt{17}, 0,1 / \sqrt{17})$ and $(-3 / \sqrt{442}, \sqrt{17 / 26},-6 \sqrt{2 / 221})$.

We will then select five "random" points on $C$. We do this by selecting five values for an axial vector scale factor $s$ and five values for an angle $\theta$ such that $0 \leq \theta \leq 2 \pi$. Our points will be of the form $u+w$ (herein $o, v, u, w, w_{1}, w_{2}$ are vectors and $s, \theta, a, b, c, d, r$ are scalars) where

$$
u=o+s v
$$




$$
w=r \cos (\theta) w_{1}+r \sin (\theta) w_{2}
$$

We now discuss recovery of a set of cylinder parameters from these five points. Given a point on $C$ we want to project orthogonally onto $L$, to get an equation involving the parameters we wish to find. As discussed above we first translate our point by subtracting $o$. We then project onto the line spanned by $v$. Subtracting this projection from the translated vector gives us our orthogonal component with length equal to the cylinder radius.

For example, one point on the cylinder in question is approximately $(5.86419,9.90186,16.3218)$. The corresponding expression we set to zero is

$$
\begin{aligned}
& a^{2} d^{2}-32.6437 a^{2} d-a^{2} r^{2}+300.791 a^{2}-2 a b c d+32.6437 a b c+11.7284 a b+19.8037 a c d- \\
& 323.233 a c-116.133 a+b^{2} c^{2}+b^{2}-19.8037 b c^{2}-19.8037 b-c^{2} r^{2}+132.435 c^{2}+11.7284 c d- \\
& 3191.429 c+d^{2}-32.6437 d-r^{2}+364.45
\end{aligned}
$$

We can use standard numerical root-finding methods to find some roots (this is, however, quite sensitive to starting points). For example, starting at $(a, b, c, d, r)=(3.2,2.8,3.7,-1.6,3.3)$, which is quite close to the values we began with, will recover those values. Starting instead at $(2.7,1.8,3.2,-.7,3)$ gives solution parameters $(a, b, c, d, r)=$ $(1.91,7.09,2.49,7.02,-4.44)$ to three decimal places.

Another well known method to find numeric roots is to sum the squares of the polynomials to be satisfied, and then minimize this sum. This too is sensitive to initial guesses. Using initial values of $(a, b, c, d, r)=$ $(2.4,1.8,2.2,-1.8,3)$ recovers the second solution shown above. Using instead $(2.4,1.8,3.2,-.8,3)$ gives a useless result with residual larger than $10^{6}$. Clearly we need a better approach.

\subsection{Solving Simultaneously for All Roots of the Cylinder Parameter Equations}

An obvious drawback to the methods seen thus far is the need for good initial guesses. We may take advantage of the fact that the equations are all polynomial and instead use a global solver suitable for such systems. We demonstrate below the utility of this approach. In order to have simpler equations for visual purposes will work with a new example comprised of integer coordinates in the range $(-10,10)$. To further simplify matters we will solve for the square of the radius (this will avoid solutions with negative values for $r$ as well as cut in half the number of complex valued solutions). An example problem with pseudorandom coordinates in the indicated range gave rise to the polynomials shown below. The points we chose to lie on the cylinder(s) are: $(7,9,8),(8,-4,-10)$, $(-4,1,4),(-9,-9,-10)$, and $(-7,-10,-10)$.

The five corresponding polynomials we set to zero are as below. To simplify notation we use $r$ to denote the square of the radius in this formulation.

$$
\begin{aligned}
& a^{2} d^{2}-16 a^{2} d-a^{2} r+113 a^{2}-2 a b c d+16 a b c+14 a b+18 a c d-144 a c-126 a+b^{2} c^{2}+b^{2}- \\
& 18 b c^{2}-18 b-c^{2} r+130 c^{2}+14 c d-112 c+d^{2}-16 d-r+145, \\
& a^{2} d^{2}+20 a^{2} d-a^{2} r+164 a^{2}-2 a b c d-20 a b c+16 a b-8 a c d-80 a c+64 a+b^{2} c^{2}+b^{2}+ \\
& 8 b c^{2}+8 b-c^{2} r+80 c^{2}+16 c d+160 c+d^{2}+20 d-r+116, \\
& a^{2} d^{2}-8 a^{2} d-a^{2} r+32 a^{2}-2 a b c d+8 a b c-8 a b+2 a c d-8 a c+8 a+b^{2} c^{2}+b^{2}-2 b c^{2}- \\
& 2 b-c^{2} r+17 c^{2}-8 c d+32 c+d^{2}-8 d-r+17, \\
& a^{2} d^{2}+20 a^{2} d-a^{2} r+181 a^{2}-2 a b c d-20 a b c-18 a b-18 a c d-180 a c-162 a+b^{2} c^{2}+b^{2}+ \\
& 18 b c^{2}+18 b-c^{2} r+162 c^{2}-18 c d-180 c+d^{2}+20 d-r+181, \\
& a^{2} d^{2}+20 a^{2} d-a^{2} r+149 a^{2}-2 a b c d-20 a b c-14 a b-20 a c d-200 a c-140 a+b^{2} c^{2}+b^{2}+ \\
& 20 b c^{2}+20 b-c^{2} r+149 c^{2}-14 c d-140 c+d^{2}+20 d-r+200
\end{aligned}
$$

In contrast to local methods, which, as we saw, may fail to get a particular solution, it turns out to be computationally straightforward to obtain all solutions to this system. We do this in Mathematica with the NSolve function. In constrast to local methods that require starting points, itt uses a hybrid symbolic-numeric technique to efficiently find all roots. Details of this technology are discussed in (Corless, 1996; Cox, 1998; Lichtblau, 2000). The idea, in short, is to compute a numeric Gröbner basis and then do an eigendecomposition of a certain matrix formed therefrom. Our solution set is as below (note again that the parameter $r$ is the square of the radius). This computation 
takes around 0.08 seconds.

$$
\begin{aligned}
& (a \rightarrow-1.03253+0.760393 i, b \rightarrow 6.11349-3.37419 i, c \rightarrow-0.322931-1.37768 i, \\
& \quad d \rightarrow-0.295427+6.8709 i, r \rightarrow 344.25+23.8554 i), \\
& (a \rightarrow-1.03253-0.760393 i, b \rightarrow 6.11349+3.37419 i, c \rightarrow-0.322931+1.37768 i, \\
& \quad d \rightarrow-0.295427-6.8709 i, r \rightarrow 344.25-23.8554 i), \\
& (a \rightarrow 0.151635, b \rightarrow-1.25748, c \rightarrow 1.58897, d \rightarrow-6.45046, r \rightarrow 83.0554), \\
& (a \rightarrow 30.9362, b \rightarrow 93.172, c \rightarrow 37.1186, d \rightarrow 92.7034, r \rightarrow 198.258), \\
& (a \rightarrow 0.613253-0.359335 i, b \rightarrow-4.49777-3.77132 i, c \rightarrow 0.102934+0.159852 i, \\
& \quad d \rightarrow-1.56979+2.23275 i, r \rightarrow 57.5606+13.7534 i), \\
& (a \rightarrow 0.613253+0.359335 i, b \rightarrow-4.49777+3.77132 i, c \rightarrow 0.102934-0.159852 i, \\
& \quad d \rightarrow-1.56979-2.23275 i, r \rightarrow 57.5606-13.7534 i)
\end{aligned}
$$

Actually we can obtain exact solutions in the same way, albeit at greater (though still quite reasonable) computational cost. This illustrates a sort of cascading hybrid algorithm: one starts with a symbolic-numeric method to handle numeric problems, then modifies it to give exact rather than approximate results.

\section{Timing[exactsolns $=$ NSolve[exprs, $\{a, b, c, d, r\}$, WorkingPrecision $\rightarrow$ Infinity]; $]$}

\subsection{Second}

The exact values for the solution set are comprised of algebraic numbers coming from defining polynomials with integer coefficients of several hundred digits.

\subsection{Overview of Other Approaches to Solving the Cylinder Equations}

We can improve considerably on the computational efficiency of finding cylinder parameters from five points. For one, a different formulation of the problem, to be utilized later, finds directions for which all points project onto the same circle in a plane perpendicular to the direction. Using this we can reduce the computational time by a substantial factor vs. the method shown above. In addition to changing the formulation of the problem to one that is computationally easier, one might also change the solver method. We discuss one very efficient alternative. This is the sparse homotopy method described in ( $\mathrm{Li}, \mathrm{Sauer}, \&$ Yorke, 1989). Here one constructs a readily solved system using information from the Newton polytope. One then forms a homotopy to move from each solution of the first system to a solution of the new system. Specifically, if we call the systems $F(x)$ and $G(x)$ respectively, where $x$ denotes a vector of variables, then one adds a new variable, $t$, and sets up the homotopy between solutions in each set as a relation $(1-t) F(x)+t G(x)=0$. At time $t=0$ we have a solution to the first system, and at time $t=1$ we have a solution to the new system. Techniques for moving along the homotopy path generally utilize a predictor-corrector method to increment $t$ by a small amount and then alter the coordinates of $x$ to maintain the relation above; a general introduction to this method is presented in (Kotsireas, 2001). For our cylinder problem there is a nice refinement that goes by the name of the "cheater's homotopy" (Li et al., 1989) wherein we start with known solutions for one set of points and hence can skip the first step of the general approach. In order to find cylinder parameters for each subsequent set of points we simply use a homotopy appropriate for the new set of equations.

An occasional disadvantage to the general sparse homotopy technique is that in some cases one has fewer actual solutions than are given by the starting system. If this occurs, then during the process of following the homotopies some must wander off to infinity. This can pose difficulties for the software: it is difficult to determine when a path is diverging, rather than following a path extending far from the initial point prior to converging to a possibly large but finite solution. For our problem family the sparse homotopy method will predict that there are eight solutions for cylinder parameters, two more than are actually present. Hence the cheater's homotopy is all the more appealing for this class of problems, since (in the generic configuration case) all paths are guaranteed to converge to finite solutions. It should be noted, however, that the general sparse method (Vershelde, Verlinden, \& Cools, 1994) is far better at approximating the correct number of solutions than prior methods based on homotopies. Moreover it tends to handle systems with far more solutions than can successfully be tackled by methods that require computation of matrix eigensystems such as that presented in (Corless, 1996). 


\subsection{The Size of the Solution Set}

The preceding example had six solutions. We now investigate further. Again taking the polynomial system (5), we form a lexicographic Gröbner basis. This is a standard tactic for computational equation manipulation (Adams \& Loustaunau, 1994; Cox, Little, \& O'Shea, 2007). The idea is that it effectively triangulates the polynomial system in a manner that will become clear below. If we order so that $a$ is the lexicographically "smallest" variable then we obtain a univariate in that variable, along with other polynomials. As the coefficients are large we will only show that first polynomial:

$$
\begin{aligned}
& 33369819849015-260250873299469 a+250872620195750 a^{2}+127385909908067 a^{3}- \\
& 186344103956650 a^{4}-259033149843189 a^{5}+8563282997415 a^{6}
\end{aligned}
$$

It is instructive to learn the structure of the Gröbner basis. The first polynomial is of degree 6 in the variable $a$ (as we already knew), and the rest are quintic in $a$ and linear and with a constant coefficient in each of the respective other variables. So now we see what was meant by triangularizing the system. To solve it one could find the six roots in $a$ and back substitute each into the remaining equations in order to get six corresponding solutions in each of the remaining variables.

This tells us to expect six solutions in general. As noted earlier this result may be found in several references. Later we will give computational proofs. For now we offer two reasons to believe this result; each may be viewed as a Monte Carlo "proof”.

1) In the theory of lexicographic Gröbner bases there is the Shape Lemma (Becker et al., 1994), which may be stated as follows. As is well known, a generic zero dimensional polynomial ideal over an infinite field is radical and in general position with respect to the last variable in any ordering of the variables. In other words, the variety has no multiplicity and moreover its finitely many points do not share any coordinates. The lemma states that under these circumstances any lexicographic Gröbner basis will have exactly one polynomial with leading term a pure product in each variable, all but the one in the smallest variable will be linear, and that one in the smallest variable will have degree equal to the size of the solution set. In addition to the Shape Lemma there is the following result: lexicographic Gröbner bases of ideals defined over rational function fields remain Gröbner bases after generic specialization of coefficients (Gianni, 1987; Kalkbrenner, 1987). In other words, there is a Zariski-open set in the parameter space for which specializations do not alter the skeleton of the basis. We use these facts as follows: if our selection of coefficients was generic, we may conclude that the generic Gröbner basis has the same shape as that of the basis we just obtained. Moreover we may believe that our selection was generic because (i) it had the correct shape of a generic basis, and (ii) we used pseudorandom data selected from a fairly large set.

2) A simulation with $2^{12}$ randomly chosen configurations always gave exactly six solutions.

One might ask why we do not simply compute a lexicographic basis for our system using indeterminates as coefficients. The answer is that it does not finish in finite time. Indeed, making just one coordinate into a parameter leads to tremendous computational effort and very large coefficients for the basis. That is to say, polynomials in that parameter are of high degree and have large integer coefficients. An alternative computational approach to finding generic cardinality of solution sets is to introduce new indeterminants as "offsets" to a given point configuration. The advantage is that one can work with a term ordering that behaves better than lexicographic orders. We anticipate further work on this approach in a future paper.

As Gröbner bases computations never leave their base field (that is, if we begin with real data then the polynomials in the basis will have real coefficients) we conclude that complex solutions will be in pairs. Thus we might have zero, two, four, or six (real) cylinders in $\mathbb{R}^{3}$. In the case of the example above we have two. We will later use the results of the large simulation tests to say a bit about percentages of examples for which one obtains given numbers of real solutions.

\section{Computational Geometry of Cylinders through Five Points}

\subsection{Finding the Implicit Equation of a Cylinder from Its Parametric Form}

Given the parameters of a cylinder, it is natural to ask how one might obtain the implicit form. The first method we show, best described as "applied brute force", is from modern elimination theory. Some references for this technique are (Adams \& Loustaunau, 1994; Cox et al., 2007; Hoffmann, 1989; Kalkbrenner, 1990). We begin with equations for $(x, y, z)$ in terms of the five parameters and the sine and cosine of an (unrestricted) angular parameter.

In more detail, we have a parametrization for the cylinder in terms of a scalar multiplier $t$ for the direction vector $v$ 
and an angle $\theta$ to determine a unit vector in the plane orthogonal to $v$. To make this parametrization algebraic we can use the usual pair of trigonometric functions, abbreviated below as algebraic variables $(c, s)$. This gives one parameter more but of course we also now have the polynomial $c^{2}+s^{2}-1$. A standard Gröbner basis method for elimination of variables uses a term ordering that is typically efficient for partially triangularizing the polynomials. In particular it weights terms that involve any of the elimination variables higher than all other terms. We form a Gröbner basis with respect to such an ordering and remove all polynomials that contain any of the elimination variables $(t, c, s\}$. What remains, a single polynomial, is the implicit relation in the variables $(x, y, z)$.

$$
\begin{aligned}
& z\left(-2 a^{2} d+2 a b c-2 d\right)+x^{2}\left(a^{2}+c^{2}\right)+a^{2} d^{2}-a^{2} r^{2}+\left(a^{2}+1\right) z^{2}+y\left(2 a c d-2 b c^{2}-2 b\right)+x(2 a b+2 c d) \\
& -2 a b c d-2 a c y z-2 a x y+b^{2} c^{2}+b^{2}-c^{2} r^{2}+\left(c^{2}+1\right) y^{2}-2 c x z+d^{2}-r^{2}
\end{aligned}
$$

Note that, as one might expect, the implicit polynomial is a function of $r^{2}$.

For the example using cylinder parameters from (4) the implicit polynomial is computed to be

$$
25 x^{2}-6 x y-8 x z+4 x+17 y^{2}-24 y z-92 y+10 z^{2}+68 z-420
$$

There is a simpler way to find the implicit form for a cylinder. Just use the formulation we described for finding the distance from a point to the axial line. This gives an equation satisfied by every point on the cylinder. Hence it will be the hypersurface expression we seek. One lesson is that brute force, while useful, is no match for finesse. The first approach remains of interest because it is a standard technique in computational algebraic geometry, and works when geometric intuition may not be so readily available.

\subsection{Finding Cylinder Parameters from the Implicit Form}

Now we look into the reverse problem of finding parameters from the implicit form. While algebraic parametrization is in general difficult, the case of quadric surfaces in $\mathbb{R}^{3}$ is not terribly hard; general methods for this are presented in chapter 5 of (Hoffmann, 1989). For the case of cylinders we will show a very simple approach which we illustrate using the example above.

As we know the general implicit form, it suffices to equate coefficients from that with those of the given numerical polynomial and then solve for the parameters. For the example with numeric parameters from (4) the numerical polynomial is provided in (7). One can equate coefficients between the symbolic implicit polynomial (6) and the numerical one (7), obtaining a straightforward system to solve. Here for example we would have $a^{2}+c^{2}=25$ as the equation resulting from the term in $x^{2}$. This system yields, as we expect, $(r \rightarrow 21, b \rightarrow 2, d \rightarrow-1, a \rightarrow 3, c \rightarrow 4)$.

Were the coefficient equations not so readily solvable we could instead do as follows. Starting with that cylinder in implicit form we generate at least five points that lie on it. To this end we might simply take values for $(x, y)$ coordinates, and solve for $z$. We then form equations for the parameters from the first five points and solve them. This gives candidate parameter values. Last we find the implicit equation corresponding to each set of parameters: the correct parameters will be the ones that recover the original implicit form (up to scalar multiple).

\subsection{Solving for Overdetermined Cylinders}

An important question to ask is what we might do to find a cylinder when we are given more than five given points? The typical case is where the points all lie approximately on a cylinder and we wish to find the best fitting one (perhaps to assess tolerance). We will use a local optimization method for this task. We can set up an expression to minimize as follows. First form the list of orthogonal complements to projections of our points onto the axial line. Then take a sum of squares of differences between projected lengths and radius.

We already saw that it is quite important to have good starting values. We do this by taking five points, solving for all exactly determined cylinder parameters therefrom, and then using other points to decide which of the six possibilities we should utilize. Specifically, at the set of "good" approximations we will have real values and our sum of squares will be near zero. This is referred to as a minimal subset method.

To illustrate we resurrect our original example but this time we use more points and we add random noise to all of them. The eight points below are thus perturbed slightly from the known example cylinder.

$$
\begin{aligned}
& (-2.61303,4.97448,-3.39489),(-6.50929,-17.4652,-19.5735),(9.39443,18.3938,18.2057), \\
& (12.7263,29.6737,32.2087),(5.481,20.4069,30.6016),(7.21938,33.4364,34.1586), \\
& (10.6382,20.9278,29.2479),(-4.81338,-25.7862,-39.1488)
\end{aligned}
$$


We first obtain a set of candidate starting values. Using the method described above, we get six possible sets. We select the best candidate by calculating values of the six implicit equations at all points, summing absolute values for each equation over all points, and using the parameters that correspond to the implicit equation that yields the smallest such sum. For a particular set of choices we obtained the residuals below.

$$
(340.514,340.514,8047.95,0.1098,1973.7,1973.7)
$$

It is clear that the fourth set of initial values is the one we should use. With this providing initial values a local minimization of the sum of squares of residuals gives the resulting parameter values below.

$$
\left\{4.0268464407121945^{* \wedge}-9,\{a \rightarrow 2.99996, b \rightarrow 1.99945, c \rightarrow 3.99999, d \rightarrow-1.00099, r \rightarrow 4.58255\}\right\}
$$

As a general remark, attempts with different optimization methods indicate that this sort of expression is quite problematic to minimize without reasonable starting points. Hence the ability to solve the exactly determined system is quite important as it provides an essential preprocessing step.

There are interesting applications to this. In the industrial realm of geometric tolerancing one wishes to measure how well an object conforms to specifications. The cylinder is of course a very common object in manufacture. A good approach to metrology involving cylinders may be found in (Devillers \& Preparata, 2000). The technology discussed therein is especially effective when the object in question is small and may be readily positioned, but one might accept a cruder approach e.g. to check an underground pipeline. For this sort of task one could probe five points, obtain from them a set of approximate cylinder parameters, then probe several others and obtain parameters for a least-squares nearest cylinder as above. One can then check whether all probed points are within specification tolerance in actual radial measure from the computed axial line. Other applications include fitting a cylinder to a point cloud (Chaperon \& Goulette, 2003; Chaperon, Goulette, \& Laurgeau, 2001), positioning of femur pieces for surgical fracture reduction (Winkelbach, Westphal, \& Goesling, 2003; Sheldon Lichtblau, private communication), and the first step of fitting peptides and other biomacromolecules to a helix (Bellesia, private communication, 2004). We note that the method above is strictly a fitting problem. If we wish to fit points in regions with multiple objects we must preprocess via image segmentation. A robust statistics approach is presented in (Roth \& Levine, 1993) that also relies on sampling exact fits of minimal subsets. In order to qualify as "robust" it requires a method to distinguish and discard outliers. Once the object points in the region are segmented one can then fit cylinders as above.

We remark that similar methods can be employed to find the smallest cylinder enclosing a given set of points. One important difference is that some applications do not necessarily have all points lying near to the cylinder surface. So taking five at random might not suffice to give a reasonable set of starting values for subseuent optimization. One way to improve on this is to cluster points into five sets, average each set, and use the resulting five points to fit cylinders to obtain a starting configuration. See (Schömer, Sellen, Teichmann, \& Yap, 2000; Brandenberg \& Theobald, 2004; Watson, 2006; Petitjean, 2012) for further discussion of this problem and its applications.

\subsection{Visualization of Cylinders Containing a Set of Points}

Once one has parameters for real cylinders containing a set of points one might wish to plot the configuration. For this purpose it is often useful to shrink the cylinder radius mildly so that the points are more readily visible. We also connect them by segments as this tends to make more clear how they are situated on the cylinder.

Let us look at an interesting configuation. Our points are $(1,0,0),(-1 / 2, \sqrt{3} / 2,0),(-1 / 2,-\sqrt{3} / 2,0),(0,0, \sqrt{2})$, and $(0,0,-\sqrt{2})$. One notes that it is hardly generic in the sense that the points form a double regular tetrahedron (with edge length of $\sqrt{3}$ ). In particular gives a pair of cylinders with axes parallel to the $y z$ coordinate plane, and this means we cannot obtain all six cylinder parameters as solutions to the equations based on (1), (3) that we have worked with thus far.

For this example we instead use the third coordinate to parametrize the cylinder axis, as $(a z+b, c z+d, z)$. With respect to this setup, one can obtain the exact solutions to the corresponding system of equations. Unlike e.g. random configurations, the exact solutions are quite readily expressed. The radii are all 9/10.

Two of the six parameter sets are

$$
\begin{gathered}
(a, b, c, d, r)=(0,1 / 10, \sqrt{2 / 3}, 0,81 / 100) \\
(a, b, c, d, r)=(-1 / \sqrt{2},-1 / 20,-1 / \sqrt{6}, \sqrt{3} / 20,81 / 100)
\end{gathered}
$$


The rest are similar to these first two but with various of the first four components negated. Here are plots using various viewpoints. We show some renditions of the solution cylinders, individually and as a set, using different graphics and viewpoints. This may help to visualize how the cylinder axes pass through pairs of faces of the doubled tetrahedra.
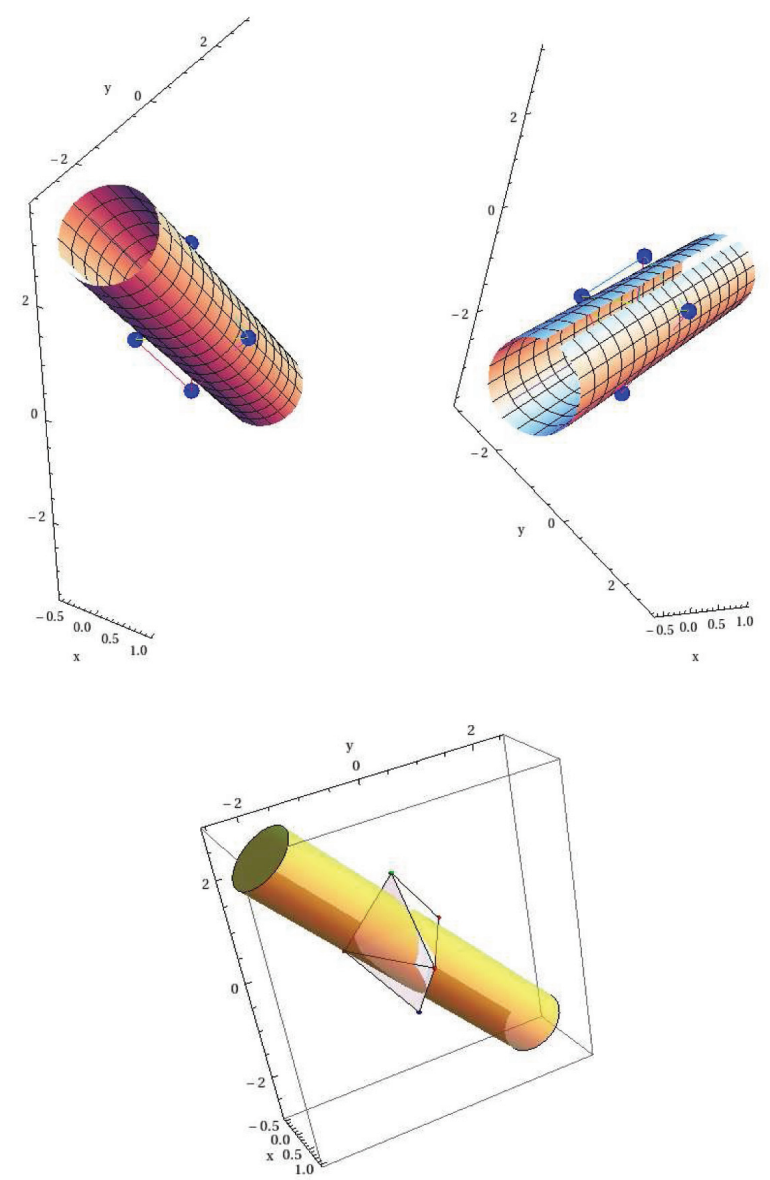

Here is a plot of all six cylinders containing these five points.

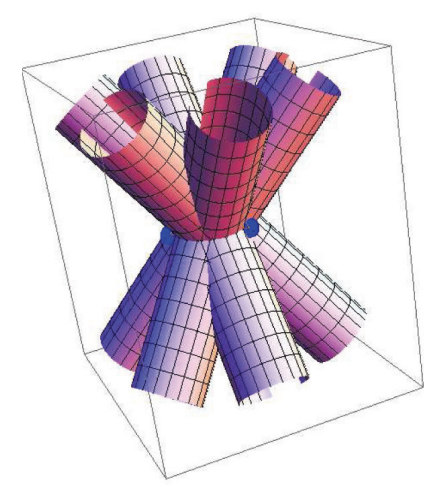

It is interesting to note that from this double regular tetrahedron construction one may obtain twelve real cylinders of a certain radius that each contain four particular points. Such an example was first presented in (Macdonald et al., 2001); here we show how it arises naturally from our construction above. We begin with a regular tetrahedron and this time glue four others onto it, one on each face. The vertices of the original tetrahedron will be our four points. Clearly from each of the glued on tetrahedra we get the six cylinders as above, each intersecting those four points and all having the same radius. While this would appear to give $4 \times 6=24$ cylinders, they pair off so that the 
actual total is twelve. We remark that computational techniques essentially identical to those we have shown can be used to find the parameters for this problem as well. A different approach, using homotopy continuation methods as described e.g. in (Li et al., 1989), was employed in (Durand, 1998). A generalization of finding cylinders of possibly different given radii through four points has been studied in (Hoffmann \& Yuan, 2000). In (Sottile, 2001) there is a construction giving six real cylinders that is similar to the one above, but using a perturbed configuration that avoids symmetry.

\section{Real Cylinders: Probabilities and Configurations}

\subsection{Enumerating Real Cylinders}

We now investigate cases in which a configuration of five points will give rise to the various possible numbers of real cylinders containing it. First we note one obvious situation for which there can be no real cylinders: if one point is inside the convex hull of the other four then, as right circular cylinders are convex, no real cylinder can contain all five points. It would be interesting to know how frequently this arises for point sets that are random under some reasonable distribution. A simple simulation is revealing. We used $2^{12}$ examples with point coordinates chosen as independent and uniformly distributed pseudorandom integers in the range $[-100,100]$. From these we found the frequencies of zero, two, four, or six real cylinders.

In one such simulation the frequencies obtained were $(931,2206,865,94)$. So roughly $23 \%$ give no real cylinders. It is natural to ask whether these are all configurations in which one point is enclosed by the other four. This turns out not to be so. We first discuss the frequency of such random configurations for which one point is enclosed by the hull of the other four. Presently we will see an open set in the configuration space for which no point lies inside the hull of the rest, and for which there are no real cylinders through all points.

To approximate the one-enclosed-by-four situation we generated $2^{14}$ random configurations and checked how many cases one point was within the convex hull of the other four. In a simulation we obtained $1147 / 2^{14}$ or about 0.070 . Thus, for the no-real-cylinder examples given a uniform distribution of points in a cube we surmise that almost three out of four cases do not arise in this way. A partly proven conjecture in (Lichtblau, 2007) states, in effect, that the remaining configurations with no real solutions may be regarded as perturbations of the ones wherein one point is enclosed by the other four.

The frequency of one point being enclosed by the others is related to some classical problems in integral geometry. One way to pose it is as a three dimensional version of Sylvester's problem (Croft, Falconer, \& Guy, 1991): What is the probability that five points chosen at random in a unit cube all lie on the convex hull they define? Another variant is to find the expected volume of a random tetrahedron in the unit cube (several other variations are posed in the reference). We will call this expected volume $v$ Tet. To see how these problems are related, we order the five random points, then ask what is the probability that the first is enclosed by the others. This is exactly that expected volume. Now observe that the expected likelihood that any one point is enclosed by the other four is $5 v T e t$, as these are each pairwise exclusive events. Indeed, by taking the average of the five cases of one-point-enclosed-by-the-rest one obtains a Monte Carlo simulation of vTet: it is in the ballpark of 1/70.

Taking this another step we might refine the estimate by quadrature. We utilized a quasi-Monte Carlo evaluation and obtained as our approximation 0.01364. This is clearly in accord with the approximation by simulation described above.

The problem of finding the expected volume of a tetrahedron with vertices independently and uniformly distributed inside a cube was recently solved (Zinoni, 2003) using an elaborate breakdown of the region and several exact multivariate integral computations. The actual value is $3977 / 216000-\pi^{2} / 2160$, or approximately .013843 . Both the quadrature result and the simulation agree with this to almost three decimal places.

\subsection{Configurations That Give Six Real Cylinders}

We previously obtained six real cylinders above by starting with a regular tetrahedron and gluing a copy of itself to one face to obtain five points. If the common face is in the $x y$ plane (so that one tetrahedron points up, the other down), then each intersects one of the three faces of the upper tetrahedron and the faces of the lower not connected by an edge to that intersected upper face. In fact it is quite clear by symmetry that if we have one real cylinder then we must have six: we get two "conjugates" by rotating, and three more by reflecting through the $x y$ plane. There is another configuration, from (Rusin), that can be seen to give six cylinders. We have four points forming vertices of a square in the $x y$ plane. This is the base of a pyramid with the fifth point as its apex above the centroid of this square. We obtain two horizontal cylinders each passing through a pair of opposite triangular faces of the pyramid. 
The remaining four each pass through a triangular face, angled upward, and an edge of the base.

We conjecture that all configurations giving rise to six cylinders in $\mathbb{R}^{3}$ are small perturbations of one of these two configurations. This idea, admittedly difficult to quantify, is based on visual experimental evidence.

\subsection{More Configurations That Give No Real Cylinders}

As noted earlier we get no real cylinders whenever one point is in the convex hull of the other four. It is also clear from experiments (and from theory in Lichtblau, 2007) that there are other configurations that give no real cylinders. We now use symbolic computation to derive a particular family of such configurations.

We begin with a double tetrahedron glued along a common face in the horizontal plane, allowing the upper vertex to vary on a vertical line. To make results of computations more concise we now work with a lower $z$ coordinate that is -1 instead of $-\sqrt{2}$. Thus our points are now $(1,0,0),(-1 / 2, \sqrt{3} / 2,0),(-1 / 2,-\sqrt{3} / 2,0),(0,0,-1)$, and $(0,0, z)$. One may readily check that when the indeterminate coordinate is 1 we have six real cylinders (all radii are 5/6). If we alter either or both of the upper and lower vertices we can jump from having six cylinders through the five points to having none. This is explained via the following symmetry argument. As noted earlier, if we have one real cylinder from such a configuration then the threefold symmetry will give us two more, for three cylinders (counting multiplicity). As this is an odd value either we must have another (and again by threefold symmetry, six altogether), or else there must be multiplicity. One can argue against multiplicity on geometric grounds, but a simple algebraic observation is that in any case we cannot have multiple solutions on more than a finite set of configurations as we move that top vertex along a vertical line (else we would have multiplicity of solutions everywhere on that variety in the configuration space). As we cannot have three real solutions counting multiplicity, we see that we either have six or none.

Below we explicitly show this phenomenon. Note that as we use a Gröbner basis approach we cannot in any straightforward way impose positivity on that mobile vertex. Were it to become negative we would have no real cylinders because either it or the other negative vertex will be in the tetrahedron hull of the remaining four vertices. Our interest, however, is in the case where it is positive, and our discussion will focus there.

We use the five equations derived from (2), with the axial parametrization $(a z+b, c z+d, z)$, to obtain the cylinder polynomials. Let us now look at a lexicographic Gröbner basis for this polynomial set. We regard the moving vertex vertical coordinate $z$ as a parameter and do the basis computation over the rational function field in that parameter. When the variables are ordered so that $c$ is lexicographically smallest we have the basis given below.

$$
\begin{aligned}
& c^{6}\left(32 z^{3}-48 z^{2}+24 z-4\right)+c^{4}\left(-48 z^{2}+48 z-12\right)+c^{2}(18 z-9)+8 z^{3}-20 z^{2}+16 z-6, \\
& a\left(-4 z^{2}+6 z-2\right)+c^{4}\left(8 z^{2}-8 z+2\right)+c^{2}(5-10 z)+2, \\
& c^{5}\left(-8 z^{2}+8 z-2\right)+c^{3}(10 z-5)+c\left(4 z^{2}-8 z+2\right)+d\left(8 z^{2}-4 z-4\right), \\
& b\left(2-8 z^{2}\right)+c^{4}\left(-8 z^{2}+8 z-2\right)+c^{2}\left(4 z^{2}+6 z-4\right)-2 z-1, \\
& r\left(-16 z^{2}-16 z-4\right)+20 z^{2}+5
\end{aligned}
$$

We saw from symmetry considerations that we get at least one real solution if and only if we get six of them. So it suffices to indicate situations where we cannot have six. For this we focus on the univariate polynomial in the last variable, $c$. First note that it is a cubic polynomial in $c^{2}$. For our task it suffices to find values of $z$ for which this cubic has no positive roots. Writing the cubic in a new variable $s=c^{2}$ we have

$$
s^{3}\left(32 z^{3}-48 z^{2}+24 z-4\right)+s^{2}\left(-48 z^{2}+48 z-12\right)+s(18 z-9)+8 z^{3}-20 z^{2}+16 z-6
$$

For $z$ sufficiently large the leading coefficient is asymptotically $32 z^{3}$. Dividing (8) by this leading coefficient we have a cubic with quadratic and linear coefficients, as rational function in $z$, asymptotically going to 0 , and “constant" term approaching $1 / 4$. That is, for $z$ sufficiently large, our cubic approaches $s^{3}+1 / 4$. As this does not have positive roots, neither does the cubic for sufficiently large $z$. Hence the sixth degree polynomial in $c$ has no real roots when $z$ is large, so the system has no real solutions in that case.

A similar but simpler argument allows us to recover a result from (Petitjean, 2012). We now allow both top and bottom vertices to vary, keeping their distances from the $x y$ coordinate plane equal. So our points are $(1,0,0)$, $(-1 / 2, \sqrt{3} / 2,0),(-1 / 2,-\sqrt{3} / 2,0),(0,0,-z)$, and $(0,0, z)$ where, as above, we assume $z$ to be positive. We set up our equations and form a Gröbner basis to solve for the cylinder parameters $(r, a, b, c, d)$ (we remind the reader 
that $r$ denotes the square of the radius) in terms of the indeterminate $z$. With that ordering of the variables the lexicographic basis is remarkably simple:

$$
\begin{aligned}
& d^{3}\left(-64 z^{4}-64 z^{2}-16\right)+3 d, \\
& c^{2}\left(1-2 z^{2}\right)+d^{2}\left(-32 z^{4}-32 z^{2}-8\right)+2, \\
& b\left(4 z^{2}+2\right)+d^{2}\left(32 z^{4}+32 z^{2}+8\right)-1, \\
& c d\left(8 z^{2}+4\right)-a, \\
& r\left(-16 z^{4}-16 z^{2}-4\right)+16 z^{4}+8 z^{2}+1
\end{aligned}
$$

Solving for the parameter $c$ will show that it is real value iff $z \geq 1 / \sqrt{2}$. The other parameters can similarly be shown to be real valued either everywhere or again exactly when $z \geq 1 / \sqrt{2}$. This is part (a) of theorem 5 from (Petitjean, 2012). Part (b) is similarly recovered by solving instead for the radius. We obtain $\frac{4 z^{2}+1}{4 z^{2}+2}$ as the common radius to all six solutions.

\section{Counting Cylinders through Five Points}

\subsection{Basic Theory and Computational Proofs}

The above investigations indicate computational ways in which one might approach questions involving cylinders through five points. We now show how purely computational methods can be brought to bear on some of the theory. Related results are presented in (Lichtblau, 2007).

Proposition 1 Generic configurations of five points in $\mathbb{R}^{3}$ lie of the surface of finitely many cylinders. Moreover an upper bound on the number of these cylinders is nine.

Proof. We set up some linear algebra similar to that already seen, but now we reduce to two equations in two variables along with the configuration parameters. The linear algebra is as follows. Without loss of generality we have one point at the origin, another at $(1,0,0)$, and a third in the $x y$ coordinate plane. We project these onto the set of planes through the origin, parametrized generically by a normal vector $(a, b, 1)$. In each such plane these three points determine a circle, and we get one equation for each of the remaining two points in order that they project onto the same circle (which is the condition that the five be cocylindrical). Our points are $(0,0,0),(1,0,0)$, $\left(x_{2}, y_{2}, 0\right),\left(x_{3}, y_{3}, z_{3}\right)$, and $\left(x_{4}, y_{4}, z_{4}\right)$. From these we obtain the polynomials below.

$$
\begin{aligned}
& \left(a^{2} b y_{2}^{2} z_{3}+a^{2} y_{2} z_{3}^{2}+a^{2} y_{2} y_{3}^{2}-a^{2} y_{2}^{2} y_{3}-2 a b^{2} x_{2} y_{2} z_{3}+a b^{2} y_{2} z_{3}+2 a b x_{2} y_{2} y_{3}-2 a b x_{3} y_{2} y_{3}-2 a x_{3} y_{2} z_{3}+a y_{2} z_{3}\right. \\
& \quad+b^{3} x_{2}^{2} z_{3}-b^{3} x_{2} z_{3}+b^{2} x_{3}^{2} y_{2}-b^{2} x_{3} y_{2}-b^{2} x_{2}^{2} y_{3}+b^{2} x_{2} y_{3}+b^{2} y_{2} z_{3}^{2}+b x_{2}^{2} z_{3}-b x_{2} z_{3} \\
& \quad+b y_{2}^{2} z_{3}-2 b y_{2} y_{3} z_{3}+x_{3}^{2} y_{2}-x_{3} y_{2}-x_{2}^{2} y_{3}+x_{2} y_{3}+y_{2} y_{3}^{2}-y_{2}^{2} y_{3}, \\
& a^{2} b y_{2}^{2} z_{4}+a^{2} y_{2} z_{4}^{2}+a^{2} y_{2} y_{4}^{2}-a^{2} y_{2}^{2} y_{4}-2 a b^{2} x_{2} y_{2} z_{4}+a b^{2} y_{2} z_{4}+2 a b x_{2} y_{2} y_{4}-2 a b x_{4} y_{2} y_{4}-2 a x_{4} y_{2} z_{4}+a y_{2} z_{4}+ \\
& b^{3} x_{2}^{2} z_{4}-b^{3} x_{2} z_{4}+b^{2} x_{4}^{2} y_{2}-b^{2} x_{4} y_{2}-b^{2} x_{2}^{2} y_{4}+b^{2} x_{2} y_{4}+b^{2} y_{2} z_{4}^{2}+b x_{2}^{2} z_{4}-b x_{2} z_{4}+ \\
& \left.b y_{2}^{2} z_{4}-2 b y_{2} y_{4} z_{4}+x_{4}^{2} y_{2}-x_{4} y_{2}-x_{2}^{2} y_{4}+x_{2} y_{4}+y_{2} y_{4}^{2}-y_{2}^{2} y_{4}\right)
\end{aligned}
$$

These are irreducible over the rationals and thus are relatively prime. So generically they have finite intersection and an upper bound is given by the Bezout theorem. In fact, as each polynomial has degree three in the variables $(a, b)$, we see that there are at most nine solutions for the cylinder axis direction parameters, hence at most nine solutions for the set of cylinder parameters.

In (Devillers et al., 2003) it is noted that this projected circles approach is related to the Delaunay triangulation of projections of the five points on all possible planes. Specifically, directions of projection where the triangulation changes are important, as these occur exactly when four points become cocircular. This gives a direct tie between the enumerative and computational geometry of cylinders through five points.

Proposition 2 Real valued solutions always have positive values for the square of the radius.

The significance of this proposition is that all real valued solutions do indeed give cylinders in $\mathbb{R}^{3}$.

Proof. Suppose we form a lexicographic Gröbner basis for the system of five generic polynomials from (3), with the radius-square variable ordered as smallest. Then generically (Shape Lemma) we have a basis containing a univariate polynomial in that variable. For each of the other variables there will correspond a linear polynomial in the basis, and it will have real valued coefficients. Suppose a solution to that univariate polynomial is real valued. 
Then the remaining cylinder parameters, on back substitution, will also be real valued as they are given by linear polynomials over the reals. Now recall that our original equations were of the form equating a sum of squares to the squared radius. Here the left hand side is a polynomial function of the input data and cylinder parameters. Hence all the original equations will have positive left hand sides, so the radius squared must also be positive.

Theorem 1 Five generic points in $\mathbb{R}^{3}$ determine six distinct sets of cylinder parameters, of which an even number (counting multiplicity) are real valued. That number can be zero, two, four, or six.

Note that the number of real valued solutions being even follows from the fact that complex solutions must appear in conjugate pairs (since all the polynomial equations have real coefficients). Moreover we saw in the enumeration simulation that all four possible cases of real valued cylinder counts arise. So we need only prove that the number of solutions is six.

Proof 1. We form a Gröbner basis with respect to a degree based term ordering for the bivariate polynomial system (9) we created in proposition 1. Inspection of the head terms reveals that there are six monomials in $(a, b)$ that are not reducible with respect to this basis and hence six solutions to the system (Corless, 1996; Cox, 1998).

Proof 2. We compute the resultant of the pair of polynomials with respect to one of the two variables. We obtain a polynomial of degree 6 in the other (with large symbolic coefficients). This means there are at most six solutions. As we already know there are at least that many, this suffices to show that there are generically six solutions.

Remark 1. One might wish to use the method of mixed volume to compute the number of solutions (Huber \& Sturmfels, 1997). One finds the convex hull of the Newton polytopes of the exponent vectors for each polynomial and then computes a mixed volume. This is easy to do using the computation from the proof of proposition 1 . Each of the two polynomials has the same set of power products in $(a, b)$ and specifically the hull of the exponent vectors is given by the vertex set $(0,0),(2,0),(2,1),(1,2)$, and $(0,3)$.

The volume of this region is 4 . The Minkowski sum of the two polytopes is just the same hull scaled to twice its size, and the mixed volume is equal to the total volume minus the sum of the volumes of each separate hull, or $16-8=8$. So the generic number of solutions for equations with these sets of exponent vectors is 8 rather than 6 . Indeed, one can verify this immediately by solving a pair of random equations that use the same power products. This shows that the cylinder through five points problem is nongeneric with respect to the theory presented in (Huber \& Sturmfels, 1997). A hint as to why this is so may be gleaned from yet another computational proof of theorem 1 presented in (Lichtblau, 2007). This sort of nongeneric example is also noted in (Huber \& Sturmfels, 1997). The related problem discussed in (Durand, 1998; Macdonald et al., 2001) similarly fails to be generic for the polyhedral homotopy solving method.

Remark 2. Proof 1 uses a brute force computation of a Gröbner basis for a system with generic configuration parameters. This approach is not tractable for many geometric problems, and that it worked here is indicative of the relative simplicity of this formulation of the problem.

Remark 3. Proof 2 is similar in method to an argument in (Schömer et al., 2000) which implies that there are at most 12 cylinders of a given radius through four fixed points.

Remark 4. Other proofs of varying levels of complexity may be found in (Bottema \& Veldkamp, 1977; Chaperon \& Goulette, 2003; Devillers et al., 2003; Lichtblau, 2007). An algorithm that effectively automates finding the cardinality of generic solution sets to geometric configuration problems will be the subject of future research. The idea would be to show that the solution count is constant in a neighborhood of a given point in configuration space.

Remark 5. In addition to the utility of the formulation (9) as a device for proving the theorem, we will mention that it is also useful for the actual compution of solution sets from five given points. While we chose to work at first with the system of five polynomials given by (3) because it was conceptually easier to formulate, numeric computations with (9) tend to be faster. The method of (Lichtblau, 2000) (e.g. as implemented in Mathematica's NSolve function) is quite effective on such bivariate polynomials. Based on published timings it appears to be competitive with methods from (Devillers et al., 2003; Durand, 1998; Petitjean, 2012). The bottleneck is in computing a degree-based Gröbner basis, as this must be done in software arithmetic at modest precision (50 or so digits suffices for this particular problem). For this formulation such a computation is quite fast even in software arithmetic. From there, the determination of actual roots can be done in hardware machine arithmetic using e.g. Lapack library code for numerical eigensystems. Using the relatively slower formulation of five variables in five unknowns we still get all solutions to a given system in around 0.1 seconds on a standard desktop machine. The formulation (9) for getting the direction parameters is around an order of magnitude faster. 


\subsection{Counting Extremal Cylinders through Four Points}

We now investigate a related problem, that of finding cylinders of extremal radii containing four specified points. The actual task of computing these can be addressed in a manner similar to that of finding cylinders through five points. For sake of brevity we forego numerical examples; methods for this are described in (Schömer et al., 2000; Brandenberg \& Theobald, 2004; Watson, 2006; Devillers et al., 2003; Petitjean, 2012). Here we will recover a known upper bound on how many such extremal cylinders can exist. The following result appears to have first been proved in (Brandenberg \& Theobald, 2004; Devillers et al., 2003).

Theorem 2 Four generic points in $\mathbb{R}^{3}$ determine at most 18 cylinders of extremal radius.

Proof. We work with a generic set of four points. After translation, rotation, and rescaling we may assume, as before, that they are $(0,0,0),(1,0,0),\left(x_{2}, y_{2}, 0\right)$, and $\left(x_{3}, y_{3}, z_{3}\right)$. We also assume no three are collinear, that is, both $y_{2}$ and $z_{3}$ are nonzero. The direction vector again will be $(a, b, 1)$. With this setup we know that any solution for the cylinder parameters must satisfy the first polynomial in (9), which we will refer to below as $p$. Moreover it is straightforward to derive the following expression for the square of the radius.

$$
\begin{array}{r}
r(a, b)=\frac{1}{4 y_{2}^{2}\left(a^{2}+b^{2}+1\right)^{2}}\left(b^{2}+1\right)\left(a^{2} y_{2}^{2}-2 a b x_{2} y_{2}+b^{2} x_{2}^{2}+x_{2}^{2}+y_{2}^{2}\right) \\
\left(a^{2} y_{2}^{2}-2 a b x_{2} y_{2}+2 a b y_{2}-2 b^{2} x_{2}+b^{2} x_{2}^{2}+b^{2}-2 x_{2}+x_{2}^{2}+y_{2}^{2}+1\right)
\end{array}
$$

We set up the extremization as a Lagrangian multiplier set of equations (a similar approach is indicated in (Schömer et al., 2000; Devillers et al., 2003). Regarding both $p$ and $r$ as functions of $(a, b)$ we obtain two equations from $\nabla r=\lambda \nabla p$. We then eliminate the Lagrange multiplier $\lambda$. Upon clearing denominators the resulting polynomial, $f$, is too large to print. It has total degree in $(a, b)$ of 9 . As our last equation, $p=0$, has degree 3 in those variables, the Bezout bound on the number of solutions for the radius squared is $3 \times 9=27$. Computing a Gröbner basis for $(p, f)$ with respect to a total degree order gives the following exponent vectors for the lead terms of the basis elements: $(2,1),(6,0),(1,6),(0,8)$. It is straightforward to verify that there are 18 monomials not reduced by these lead terms, and hence 18 solutions to the system.

In (Devillers et al., 2003) it is conjectured that there are at most 12 distinct real solutions for the radius. They indicate an example where there are 18 such counting multiplicity, but only 12 distinct values; perhaps more importantly, six of the solutions do not have real-valued axial direction vectors $s$. Perhaps surprisingly, it turns out that one can obtain (at least) 14 real cylinders. This situation arises with the four points $(0,0,0),(1,0,0)$, $(1 / 2, \sqrt{3} / 2,0),\left(2 / 5,1 /(2 \sqrt{3}), \sqrt{\frac{2}{3}}-1 / 100\right)$. This may be veriffied as follows. A straightforward computation shows that there are 14 real solutions for radius and direction vector parameters. After that one can use a resultant to eliminate the parameter $a$ between $f$ and the radius equation (10), obtaining a polynomial relation that defines $r$ implicitly as a function of $b$. We know (and also can verify numerically) that $r^{\prime}(b)$ vanishes at each solution. Moreover one can show that $r^{\prime \prime}(b)$ is negative at 7 solutions and positive at seven others, and that, as must be the case, they are interleaved on the two real components of the vanishing set of $f(a, b)$.

We provide numerical approximations to the 14 sets of parameter values for the set of locally extremal cylinders containing the given points. Each is shown as $r^{2}$, direction, offset.

$$
\begin{array}{lll}
0.239454, & (-1.52114,-0.433428,1), & (0.73789,0.428604,0) \\
0.243633, & (-1.13822,-0.991331,1), & (0.743623,0.428094,0) \\
0.248353, & (-0.181966,1.56532,1), & (0.504142,-0.0501668,0) \\
0.248454, & (-0.150935,1.34794,1), & (0.4974,0.0359956,0) \\
0.248473, & (-0.136877,1.41104,1), & (0.499396,0.00934622,0) \\
0.249933, & (-0.0283518,1.41415,1), & (0.499995,0.000374409,0) \\
0.26184, & (1.61489,-1.10319,1), & (0.0413145,0.570812,0) \\
0.266748, & (0.732818,-0.505806,1), & (0.399877,0.339226,0) \\
0.271896, & (6.54262,-9.98216,1), & (-1.52227,3.11637,0) \\
0.272064, & (-0.694179,0.433211,1), & (0.415297,0.334521,0) \\
0.280267, & (8.03646,-0.714325,1), & (-1.94765,0.643932,0)
\end{array}
$$




$\begin{array}{lll}0.282078, & (0.0638058,-0.693965,1), & (0.493415,0.220321,0) \\ 0.290591, & (9.45907,17.8707,1), & (-2.41353,-5.52167,0) \\ 0.290792, & (0.538069,0.274628,1), & (0.543646,0.317641,0)\end{array}$

\subsection{Counting Cylinders of Given Radius through Four Points}

We mentioned at the end of section 3 that given four points in space and a fixed radius $r$, there can be at least 12 cylinders of that radius containg the points. It has been proven in (Devillers et al., 2003; Macdonald et al., 2001). We give a computational proof below, similar to those used in the preceding proofs.

Theorem 3 Four generic points in $\mathbb{R}^{3}$ and a given radius determine 12 (complex) cylinders.

We reiterate that this gives the generic count of the number of complex solutions; the number of real solutions can be strictly smaller.

Proof. Again we work with points $(0,0,0),(1,0,0),\left(x_{2}, y_{2}, 0\right)$, and $\left(x_{3}, y_{3}, z_{3}\right)$ and direction vector $(a, b, 1)$. Again this gives the first polynomial in $(0)$ and also equation (0) for the square of the radius. This now is a fixed value, hence may be treated as a parameter. We clear denominators and compute a resultant to eliminate a from the two polynomials. This gives a polynomial of degree $12 \mathrm{in} \mathrm{b.} \mathrm{Hence} \mathrm{there} \mathrm{are} \mathrm{at} \mathrm{most} 12$ solutions. As simple examples show there must be at least 12 , this is the generic solution count.

In the preceding example of four points containing 14 extremal cylinders, we remark that one locally minimal radius is larger than one local maximum. This happens of necessityinsofar as otherwise we would have a violation of theorem 3. Indeed, the extrema must be interleaved on the curve in $(a, b)$ space of direction vector parameters. Thus were all minima smaller than all maxima, then all radius values in the interval between the largest minimum and smallest maximum would correspond to 14 cylinders containing the four points of that example.

\section{Nongeneric Solution Sets}

Some further problems of interest include understanding the configurations of five distinct points that are degenerate for the problem at hand. Specifically we would like to know:

(1) When the number of solutions is infinite.

(2) When the number, counting multiplicity, is strictly less than 6 .

(3) When there are multiple solutions.

Question (2) and some aspects of (1) are addressed in (Lichtblau, 2007). Among other things we note that a sufficient condition to have either infinitely many cylinders through five points, or at most four, is that the points be coplanar. It is conjectured in (Lichtblau, 2007) that these are also necessary conditions, and moreover that infinitely many real cylinders exist exactly when either four points are collinear or three are collinear with the line determined by the remaining two parallel to the line through those first three. This is also discussed in (Petitjean, 2008). Note that the assumption of real values is necessary: (Lichtblau, 2007) gives a counterexample wherein there are infinitely many complex valued solutions to the cylinder equations for a given set of five points in complex space that do not satisfy the collinearity hypotheses.

Clearly the two defining cubics of (9) are distinct (since the vertices are distinct by assumption). We proceed to show that they do not share a common factor unless they are coplanar and al least three are collinear.

Proposition 3 Assume we have five distinct points in $\mathbb{R}^{3}$, three of our points are collinear. Then there are infinitely many real solutions if and only if the either the remaining two determine a line parallel to the first three or else one of the remaining two is on the line through the first three.

Proof. It is straightforward to show that either four collinear points, or three collinear points and two others on a parallel line, give an infinite solution set. We now show the converse.

For reasons provided earlier, without loss of generality our points are $(0,0,0),(1,0,0),\left(x_{2}, 0,0\right),\left(x_{3}, y_{3}, z_{3}\right)$, and $\left(x_{4}, y_{4}, z_{4}\right)$. Since we have three points on the $x$ axis, and require real cylinders, the direction vector must be $(1,0,0)$. We use the formulation from (3), this time with our direction vector fixed as above. Solving for the offset vector parameters, the radius squared, and two coordinates $y_{3}$ and $z_{3}$, we have three cases.

(1) $y_{3}=z_{3}$. In this case four points are coplanar. Moreover $(b, d, r)$ are not determined so we have an infinite solution set.

(2) $y_{3}=y_{4}$ and $z_{3}=z_{4}$. In this case the line between the last two is parallel to that between the first three. Again 
$(b, d, r)$ are not determined and we have an infinite solution set.

(3) We have a solution for $(b, d, r)$ with each having a denominator (which cannot vanish) of $y_{4} z_{3}-y_{3} z_{4}$. This forces the line between the last two points to be noncollinear with that between the first three, and moreover neither point can lie on the $x$ axis, so no four points are collinear.

Taken together, these cases prove the proposition.

With this special case out of the way we can now prove the general result, that five points, with no three collinear, cannot have an infinite solution set for the cylinder equations.

Theorem 4 Assume we have five distinct points in $\mathbb{R}^{3}$ with no three collinear. Then there are finitely solutions to the cylinder equations.

Proof. If the solution set is infinite then these polynomials share a common factor, call it $g(a, b)$. In forming the system (9) we are free to select any three of the five given points as our first three (they form a triangle that is a base to two tetrahedra, each formed by that base and one of the remaining two points). Next observe that since the solution set is infinite, every such system must have a common factor. Hence there is a nontrivial factor of $g(a, b)$ that divides each polynomial in every such system.

We recall from (Devillers et al., 2004; Lichtblau, 2007) that the polynomial system (9) has three solutions at infinity. These arise as the directions of the lines connecting any pair of the first three points, that is, as directions of the base triangle. Each such base triangle gives a set of three possible solutions at infinity. It is a general result (Cox et al., 2007) that the variety for $g(a, b)$ must intersect the line at infinity in projective space. Thus there must be a point at infinity common to the polynomial systems given by every choice of base triangle. By the assumption that no three points are collinear, no such direction is shared between any pair of base triangles, hence there can be no point at infinity. This contradiction proves the theorem.

We remark that in order to have a common edge to all the base triangles, collinearity of three points alone does not suffice. It can be seen that either four collinear points, or three collinear points and two others forming a line parallel thereto, is required. This is another way to show proposition 3.

A related matter of interest is to describe the configurations that give some specified number (even, counting by multiplicity) of real solutions. In (Lichtblau, 2007) there is considerable discussion of the case of no real cylinders. Again one might wish to approach this computationally using discriminant variety tools from (Lazard \& Rouillier, 2007). Here the ideal of interest is the set of certain Jacobian minors (as well as the original polynomials). At multiple solutions these will vanish. Hence any characterization of these, intersected with real space, will include the boundaries in the configuration space between different numbers of real solutions. Computationally this would appear to be a daunting problem and it would be interesting to see if any existing software can make progress with it. Also as the result is expressed in terms of algebraic relations, it would then be useful to understand from them the underlying geometric relations that describe the four cases of real solution cardinalities.

\section{Summary}

We have discussed computational methods for finding cylinders through a given set of five points in $\mathbb{R}^{3}$. Along the way we have covered several related problems and computational approaches thereto. We have investigated various real valued scenarios using simulation. We used computational methods to prove results of an enumerative nature. Overall we have combined geometric reasoning with Gröbner bases and several related tools from symbolic computation in order to study a rich family of problems from enumerative and computational geometry.

\section{Acknowledgements}

I am pleased to acknowledge helpful conversations with Dick Bishop, John D'Angelo, Dan Grayson, and Dave Rusin. I thank David Cox and Thorsten Theobald for bringing to my attention the earlier work of Bottema and Veldkamp. I thank Jack Wetzel for inviting me to speak twice on this topic at the UIUC weekly Geometry seminar. I thank an anonymous and very patient referee for carefully reading an earlier draft and making several detailed comments and suggestions that helped to clarify, and in some cases repair, various points in the exposition. I am grateful to an anonymous referee of the present article for providing helpful comments and critique, for bringing to my attention the recent article by Petitjean (2012), and for suggesting that a related problem, the enumeration of extremal cylinders through four points, also warranted consideration herein. This moreover led to correspondence with Michel Petitjean that was also quite helpful during the revision process. 


\section{References}

Adams, W., \& Loustaunau, P. (1994). An Introduction to Gröbner Bases. Graduate Studies in Mathematics 3. American Mathematical Society.

Becker, E., Marinari, M. G., Mora, T., \& Traverso, C. (1994). The shape of the Shape Lemma. In Proceedings of the 1994 International Symposium on Symbolic and Algebraic Computation (ISSAC 94), 129-133. ACM Press. http://dx.doi.org/10.1145/190347.190382

Bellesia, G. (2004). Private communication.

Bottema, O., \& Veldkamp, G. (1977). On the lines in space with equal distances to $n$ given points. Geometriae Dedicata, 6, 121-129. D. Reidel Publishing Company. http://dx.doi.org/10.1007/BF00181587

Brandenberg, R., \& Theobald, T. (2004). Algebraic methods for computing smallest enclosing and circumscribing cylinders of simplices. Applicable Algebra in Engineering, Communication and Computation, 14, 439-460. http://dx.doi.org/10.1007/s00200-003-0146-0

Buchberger, B. (1998). Applications of Gröbner bases in non-linear computational geometry. In R. Janssen (Ed.), Proceedings of the International Symposium on Trends in Computer Algebra, 87, Lecture Notes in Computer Science, 296, 52-80. Springer-Verlag. http://dx.doi.org/10.1007/3-540-18928-9

Chaperon, T., \& Goulette, F. (2003). A note on the construction of right circular cylinders through five 3D points. Technical Report, Centre de Robotique, Ecole des Mines de Paris.

Chaperon, T., Goulette, F., \& Laurgeau, C. (2001). Extracting cylinders in full 3D data using a random sampling method and the Gaussian image. In Ertl, T., Girod, B., Greiner, G., Niemann, H., \& Seidel, H-P. (Eds.), Proceedings of the of the 6th International Fall Workshop Vision, Modeling, and Visualization (VMV 01), Stuttgart, Germany, November 2001, 35-42.

Corless, R. (1996). Editor's corner: Gröbner bases and matrix eigenproblems. ACM Sigsam Bulletin: Communications in Computer Algebra, 30(4), 26-32. http://dx.doi.org/10.1145/242961.242968

Cox, D. (1998). Introduction to Gröbner bases. In Cox, D. \& Sturmfels, B. (Eds.), Proceedings of Symposia in Applied Mathematics, 53, 1-24, ACM Press.

Cox, D., Little, J., \& O'Shea, D. (2007). Ideals, Varieties, and Algorithms: An Introduction to Computational Algebraic Geometry and Computer Algebra. Undergraduate Texts in Mathematics. Springer-Verlag.

Croft, H. T., Falconer, K., \& Guy, R. T. (1991). Unsolved Problems in Geometry. 54-55. Springer-Verlag. http://dx.doi.org/10.2307/2324169

Devillers, O., Mourrain, B., Preparata, F., \& Trebuchet, P. (2003). On circular cylinders by four or five points in space. Discrete and Computational Geometry, 28, 83-104. http://dx.doi.org/10.1007/s00454-002-2811-7

Devillers, O., \& Preparata, F. (2000). Evaluating the cylindricity of a nominally cylindrical point set. Symposium on Discrete Algorithms (SODA 2000), 518-527.

Durand, C. (1998). Symbolic and Numerical Techniques for Constraint Solving. Ph.D thesis. Purdue University, Department of Computer Science.

Gianni, P. (1987). Properties of Gröbner bases under specialization. In Davenport, J. (Ed.), European Converence on Computer Algebra (Eurocal 87), Lecture Notes in Computer Science, 378, 293-297. Springer-Verlag. http://dx.doi.org/10.1007/3-540-51517-8

Hoffmann, C. (1989). Geometric and Solid Modelling: An Introduction. Morgan Kaufmann.

Hoffmann, C. M., \& Yuan, B. (2001). On spatial constraint solving approaches. In Richter-Gebert, J. \& Wang, D. (Eds.), Proceedings of the Third International Workshop on Automated Deduction in Geometry (ADG 2000). Lecture Notes in Computer Science, 2061, 1-15. Springer-Verlag. http://dx.doi.org/10.1007/3-540-45410-1_1. See also: There are 12 common tangents to four spheres, 2000. Retrieved from http://www.cs.purdue.edu/homes/cmh/distribution/SphereTangents.htm http://dx.doi.org/10.1007/BF02770870

Huber, B., \& Sturmfels, B. (1997). Bernstein's theorem in affine space. Discrete and Computational Geometry, 17, 137-141. http://dx.doi.org/10.1007/BF02770870 
Kalkbrenner, M. (1987). Solving systems of algebraic equations by using Gröbner bases. In Davenport, J. (Ed.), European Conference on Computer Algebra (Eurocal 87). Lecture Notes in Computer Science, 378, $282-292$. Springer-Verlag. http://dx.doi.org/10.1007/3-540-51517-8

Kalkbrenner, M. (1990). Implicitization of rational parametric curves and surfaces. In Sakata, S. (Ed.), Applied Algebra, Algebraic Algorithms, and Error-Correcting Codes (AAECC8). Lecture Notes in Computer Science, 508, 249-259. Springer-Verlag. http://dx.doi.org/10.1007/3-540-54195-0_55

Kotsireas, I. (2001). Homotopies and polynomial system solving I: Basic principles. A CM Sigsam Bulletin: Communications in Computer Algebra, 35(1), 19-32. http://dx.doi.org/10.1145/504331.504334

Lazard, D., \& Rouillier, F. (2007). Solving parametric polynomial systems. Journal of Symbolic Computation, 42(6), 636-667. http://dx.doi.org/10.1016/j.jsc.2007.01.007

Lichtblau, D. (2000). Solving finite algebraic systems using numeric Gröbner bases and eigenvalues. In Torres, M., Molero, J., Kurihara, Y., \& David, A. (Eds.), Proceedings of the World Conference on Systemics, Cybernetics, and Informatics (SCI 2000), 10, 555-560. (Concepts and Applications of Systemics, Cybernetics, and Informatics), International Institute of Informatics and Systemics.

Lichtblau, D. (2007). Cylinders through five points: complex and real enumerative geometry. In Botana, F. \& Recio, T. (Eds.), Proceedings of the Sixth International Workshop on Automated Deduction in Geometry (ADG 2006), Lecture Notes in Artificial Intelligence, 4869, 80-97. Springer-Verlag. doi:10.1007/978-3-540-77356-6_6

Li, T. Y., Sauer, T., \& Yorke, J. A. (1989). The cheater's homotopy: an efficient procedure for solving systems of polynomial equations. SIAM Journal on Numerical Analysis, 26(5), 1241-1251. http://dx.doi.org/10.1137/0726069

Macdonald, I. G., Pach, J., \& Theobald, T. (2001). Common tangents to four unit balls in $\mathbb{R}^{3}$. Discrete and Computational Geometry, 26(1), 1-17.

Petitjean, M. (2012). About the algebraic solutions of smallest enclosing cylinders problems. Applicable Algebra in Engineering, Communication and Computation. http://dx.doi.org/10.1007/s00200-012-0171-y

Roth, G., \& Levine, M. D. (1993). Extracting geometric primitives. Computer Vision, Graphics and Image Processing: Image Understanding, 58(1), 1-22. http://dx.doi.org/10.1006/cviu.1993.1029

Rusin, D. Retrieved from http://www.math-atlas.org/98/5pt.cyl

Schömer, E., Sellen, J., Teichmann, M., \& Yap, C. (2000). Smallest enclosing cylinders. Algorithmica, 27, 170186. http://dx.doi.org/10.1145/237218.237412

Sottile, F. (2001). From enumerative geometry to solving systems of polynomial equations with Macaulay 2 . In Eisenbud, D., Grayson, D., Stillman, M., \& Sturmfels, B. (Eds.), Computations in Algebraic Geometry with Macaulay, 2, Algorithms and Computation in Mathematics, 8, 101-129. Springer-Verlag.

Vershelde, J., Verlinden, P., \& Cools, R. (1994). Homotopies exploiting Newton polytopes for solving sparse polynomial systems. SIAM Journal on Numerical. Analysis, 31(3), 915-930. http://dx.doi.org/10.1137/0731049

Watson, G. A. (2006). Fitting enclosing cylinders to data in $\mathbb{R}^{n}$. Journal of Numerical Algorithms, 43(2), $189-196$. http://dx.doi.org/10.1007/s11075-006-9054-2

Winkelbach, S., Westphal, R., \& Goesling, T. (2003). Pose estimation of cylindrical fragments for semi-automatic bone fracture reduction. In Michaelis, B., \& Krell, G. (Eds.), Pattern Recognition (DAGM 2003), Lecture Notes in Computer Science, 2781, 566-573. Springer-Verlag.

Wolfram Research, Inc. (2010). Champaign, Illinois. Mathematica, 8. Retrieved from http://www.wolfram.com

Zinani, A. (2003). The expected volume of a tetrahedron whose vertices are chosen at random in the interior of a cube. Monatshefte für Mathematik, 139, 341-348. http://dx.doi.org/10.1007/s00605-002-0531-y 